\title{
Cellular senescence in human mammary epithelial cells (HMEC) is accompanied by an increase of elastin formation C Bertram* and R Hass
}

\begin{abstract}
Address: Medizinische Hochschule Hannover, Dept. of Gynecology, AG Biochemistry and Tumor Biology, Hannover, Germany
\end{abstract}
* Corresponding author

from 12th Joint Meeting of the Signal Transduction Society (STS). Signal Transduction: Receptors, Mediators and Genes Weimar, Germany. 29-31 October 2008

Published: 26 February 2009

Cell Communication and Signaling 2009, 7(Suppl I):A56 doi:I0.II86/I478-8IIX-7-SI-A56

This abstract is available from: http://www.biosignaling.com/content/7/SI/A56

(c) 2009 Bertram and Hass; licensee BioMed Central Ltd.

Normal human mammary epithelial cells (HMEC) have a finite life span, regulated by a process termed cellular senescence. After an initial stress-associated senescence barrier, some cells are subjected to a self-selection process triggered by the loss of the $G_{1}$ cell cycle regulator p $16^{\text {INK4a }}$ expression. This effect is associated with an escape from the growth plateau to resume cell cycle progression and to undergo further cell divisions. Thereafter, these so-called "post-selection" HMEC encounter a second senescence barrier, termed agonescence. Our recent work using an siRNA approach revealed an essential impact of the extracellular matrix metalloproteinase-7 (MMP-7) on the aging process of post-selection HMEC. Since MMP-7 is involved in a variety of cellular mechanisms, including processing of inactive precursor proteins, ectodomain shedding of extracellular signaling molecules and remodeling of the extracellular matrix (ECM), respectively, we could observe considerable alterations of the ECM during senescence of HMEC. Thus, the formation of characteristic structures similar to elastin was detectable, which markedly increased in agonescent HMEC cultures after passage 15. Moreover, Western blot analysis revealed an up-regulated expression of tropoelastin, the soluble precursor of the ECM protein elastin, during the aging process of HMEC. This was paralleled by an enhanced activity of the enzyme lysyl oxidase (LOX) which is responsible for cross-linking tropoelastin monomers to form insoluble elastic fibers. Thus, enhanced LOX activity was detectable in cellular homogenates as well as in the culture supernatant during HMEC aging between passage 12 up to passage 16 . Together with the down-regulation of MMP-7, the increased formation of elastic fibers supported an essen- tial effect of distinct ECM components and the microenvironment on HMEC aging.[1]

\section{References}

I. Bertram C, Hass R: MMP-7 is involved in aging of primary human mammary epithelial cells (HMEC). Exp Gerontol 2008, 43:209-217. 